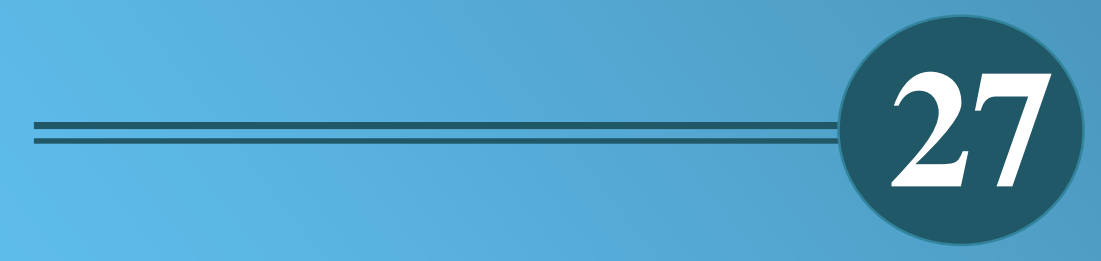

\title{
SISTEMA DE MONITOREO DE EMBARCACIONES DE PESCA ARTESANAL DE ANCONCITO, PROVINCIA DE SANTA ELENA, ECUADOR, UTILIZANDO TECNOLOGÍA AIS - AUTOMATIC IDENTIFICATION SYSTEM.
}

Ashley Avilés Bastidas, Germán Sánchez Jordán, Freddy Villao Quezada 


\title{
Sistema de monitoreo de embarcaciones de pesca artesanal de Anconcito, provincia de Santa Elena, Ecuador, utilizando tecnología AIS - Automatic Identification System
}

\author{
Monitoring system of artisanal fishing boats of Anconcito, Santa Elena province, \\ Ecuador, using AIS technology - Automatic Identification System
}

\author{
Ashley Avilés Bastidas, Germán Sánchez Jordán, Freddy Villao Quezada \\ Facultad de Ingeniería Eléctrica y Computación (FIEC) \\ Escuela Superior Politécnica del Litoral (ESPOL) \\ ashkaabi@espol.edu.ec; gerlesan@espol.edu.ec; fvillao@espol.edu.ec
}

\begin{abstract}
Resumen
Ante la evidente crisis que se está presentando en Ecuador debido al incremento en los índices de robos en altamar, principalmente de los motores fuera de borda de las lanchas de pesca artesanal, es necesario implementar un sistema de control eficaz que ofrezca seguridad para la comunidad pesquera; con este fin se propone un sistema de detección de embarcaciones de pesca artesanal con tecnología AIS. En este estudio se explican todos los beneficios que ofrece esta tecnología que actualmente se usa para el monitoreo de buques, así como las aplicaciones para un sistema de monitoreo de embarcaciones pequeñas, las clases de equipos disponibles y sus principales características. Con este objetivo se propone el diseño de una red que permita ubicar las embarcaciones de la comunidad pesquera de Anconcito, que es una de las zonas más afectadas por la delincuencia. Además, se anticipan los resultados esperados con este diseño y varias alternativas técnicas para mejorar la cobertura del sistema.
\end{abstract}

Palabras Clave: AIS, Anconcito, faro, monitoreo, pesca, SOTDMA.

\begin{abstract}
Given the evident crisis that is occurring in Ecuador due to the increase in the rates of robberies on the high seas, mainly of the outboard motorboats of the artisanal fishing boats, it is necessary to implement an effective control system that provides security for the fishing community. To this end, a system of detection of artisanal fishing vessels with AIS technology is proposed. In this study, all the benefits of this technology are explained which is currently used for monitoring ships, as well as the applications for a monitoring system of small boats, the kinds of equipment available and their main characteristics. With this objective, the design of a network that allows locate the vessels of the fishing community of Anconcito, which is one of the areas most affected by crime, is proposed. In addition, the expected results with this design and several technical alternatives to improve the coverage of the system are anticipated.
\end{abstract}

Keywords: AIS, Anconcito, beacon, monitoring, fishing, SOTDMA. 


\section{Introducción}

En Ecuador, la actividad agrícola, ganadera y pesquera representa el 7\% del PIB (según el INEC en el 2010); sin embargo, en algunos pueblos costeros la pesca artesanal es la principal fuente de ingresos.

Santa Elena y Esmeraldas son las provincias con mayor índice de delitos de robo de motores fuera de borda; en la provincia primeramente nombrada se encuentra el $21.4 \%$ del total de pescadores a nivel nacional.

\subsection{Planteamiento del problema}

El robo a embarcaciones de pesca artesanal es un problema que afecta directa e indirectamente a la población ecuatoriana, especialmente a los pueblos costeros. Cuando a una embarcación le roban el motor fuera de borda y/o los implementos de trabajo, ésta queda fuera de operación lo cual implica una disminución en la actividad económica; esto no solamente afecta al dueño de la embarcación y su familia, sino a la economía del sector pesquero, en general.

No solamente la economía se ve afectada por el hampa, la peor consecuencia de estos atracos es la pérdida de vidas humanas. Al ser atacados los pescadores a varias millas de la costa y ser despojados de su motor fuera de borda y equipos de comunicación, quedan a la deriva e incomunicados. El patrullaje de los guardacostas y reportes de desaparición por parte de los familiares de los pescadores ayudan a encontrar y rescatar en ocasiones a los pescadores, pero existen casos fatales en los que no se da el aviso a tiempo y quedan como desaparecidos.

En los últimos años ha aumentado el índice de delincuencia en altamar sobre todo a los botes pesqueros artesanales ya que, al no contar con un sistema de seguridad apropiado se vuelven presa fácil para el hampa.

Según la Dirección Nacional de Espacios Acuáticos lo que más se reporta como robado en estos atracos son los motores fuera de borda, cuyo valor, nuevo, varía entre los $\$ 6000$ y $\$ 12000$ y que, generalmente son vendidos en Perú como de segunda mano en aproximadamente \$2000. A continuación, se muestra una tabla del número de motores reportados como robados en el período 2011-2015:

Tabla 1: Número de motores reportados como robados a nivel nacional

\begin{tabular}{c|c}
\hline AÑ & $\begin{array}{c}\text { NÚMERO DE } \\
\text { MOTORES }\end{array}$ \\
\hline 2011 & 291 \\
2012 & 292 \\
2013 & 399 \\
2014 & 224 \\
2015 & 281
\end{tabular}

Fuente: DNEA

\subsection{Delimitación de la zona}

Debido a los constantes atracos reportados en la zona de Anconcito y a los estudios realizados por las autoridades pertinentes, se ha determinado que la zona de cobertura del sistema abarque principalmente el frente ChanduyAnconcito, que es uno de los sectores críticos que se observan en la gráfica de georreferenciación mostrada en la figura 1.

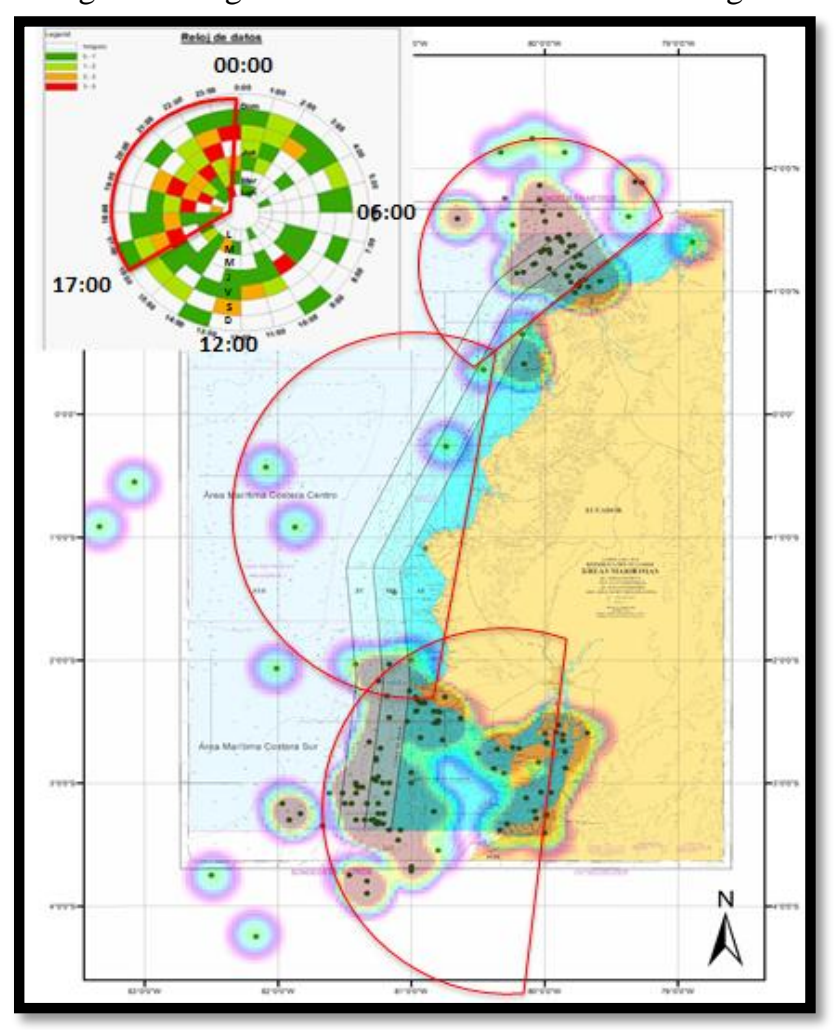

Figura 1. Mapa de georreferenciación de robos de motores en el 2014-2015

Se propone un sistema con un alcance de $20 \mathrm{mn}$ que dé cobertura en el frente Chanduy-Anconcito, extendiéndose hasta la puntilla de Santa Elena.

\subsection{Tecnología AIS}

El Sistema de Identificación Automática (AIS - Automatic Identification System) es un sistema de ayuda a la navegación, diseñado originalmente para la localización de buques con el propósito de asistirlos para evitar colisiones. Con el paso del tiempo se fueron adicionando características de interés al sistema.

AIS es un sistema abierto, esto es que las señales transmitidas no tienen ningún tipo de cifrado y cualquier equipo receptor AIS puede acceder a la información. Sin embargo, se han implementado algoritmos que permiten cifrar la información en casos de seguridad nacional o comunicaciones militares.

AIS utiliza la banda VHF, específicamente los canales 87B, también llamado AIS1, correspondiente a la frecuencia 161.975 MHz y 88B, también llamado AIS2, correspondiente a la frecuencia $162.025 \mathrm{MHz}$ [1]. Ambos canales son full dúplex y son reservados a nivel mundial para comunicaciones 
marítimas de este tipo. El alcance es como cualquier sistema VHF, depende de la altura de las antenas, potencia de salida del transmisor y las condiciones de propagación; no necesita línea de vista por lo que en mar abierto el alcance promedio es de entre 20 y 30 millas náuticas.

En general, el sistema consta de estaciones móviles y estaciones fijas; las estaciones móviles son transceivers que constan de al menos un transmisor y un receptor para comunicación bidireccional, mientras que las estaciones fijas pueden tener un transpondedor, con un transmisor y un receptor para comunicación bidireccional, o solo un equipo receptor para comunicación unidireccional.

Existen estaciones AIS que son específicamente de ayuda a la navegación y se colocan en boyas, faros o balizas; estos dispositivos emiten un tipo de mensaje especial con información detallada de la ayuda a la navegación que tanto la autoridad de Señalización Marítima y los buques necesitan.

Hay equipos que traen incorporados un panel LCD y otros un puerto de salida para conectar un display compatible, en esta pantalla se muestra información sobre las estaciones visibles dentro del rango: datos estáticos, dinámicos, de navegación y mensajes cortos relativos a la seguridad. Esta información se actualiza automáticamente y dependiendo del equipo, el periodo de tiempo puede ser configurado, inclusive para cada tipo de información.

La información AIS transmitida por un barco incluye cuatro tipos diferentes de información: estática, dinámica, relativa al viaje y textos y mensajes.

\section{a) Información estática}

Datos básicos de los buques como el nombre, MMSI, distintivo, número OMI, dimensiones y tipo de barco. Generalmente esta información se transmite cada 6 minutos.

b) Información dinámica

Datos de posición del buque como la posición, el rumbo efectivo, la velocidad efectiva, el rumbo verdadero y la condición de navegación. Dependiendo de las condiciones se actualiza de forma continua.

c) Información relativa al viaje

Datos requeridos para el control de tráfico VTS como carga peligrosa (en caso de llevarla), destino, ETA y plan de ruta. Al igual que la información estática es transmitida cada 6 minutos.

d) Textos y mensajes

Mensajes cortos relativos a la seguridad del buque o a la navegación [2].

AIS1 y AIS2 son los únicos canales para la comunicación de equipos AIS por lo que es necesario un sistema de compartición de tiempo para evitar interferencias y colisiones. AIS utiliza un esquema de compartición denominado SOTDMA (Self Organized Time Division
Multiple Access) que es un esquema de acceso que provee un enlace central para funcionamiento autónomo de la red en altamar. Este esquema de compartición de tiempo divide cada unidad de tiempo (minuto) en 2250 slots o ranuras usadas para hacer las transmisiones de diferentes estaciones dentro de un mismo rango; al haber disponible dos canales para la comunicación AIS existen 4500 ranuras o slots disponibles en total por unidad de tiempo.

Para que SOTDMA funcione adecuadamente, todas las estaciones tienen una referencia de tiempo en común, que es obtenida mediante GPS, para asegurar la correcta sincronización y que cada estación pueda determinar de forma precisa el inicio de cada slot. Cada transmisión incluye información del slot que será usado para la siguiente transmisión, esto permite a los receptores construir un mapa de que slots utiliza cada estación. Este mapa permite a las estaciones evitar usar slots "reservados" por otras estaciones y así evitar superposiciones. Las estaciones móviles puedes trasladarse de un área o "celda" a otra donde haya otras estaciones con un mapa de slots diferentes, esto implica que se deba modificar el slot a usar; generando entonces un sistema dinámico y auto-organizado en tiempo y espacio [3]. En la figura 2 se observa un diagrama del esquema de compartición de SOTDMA.

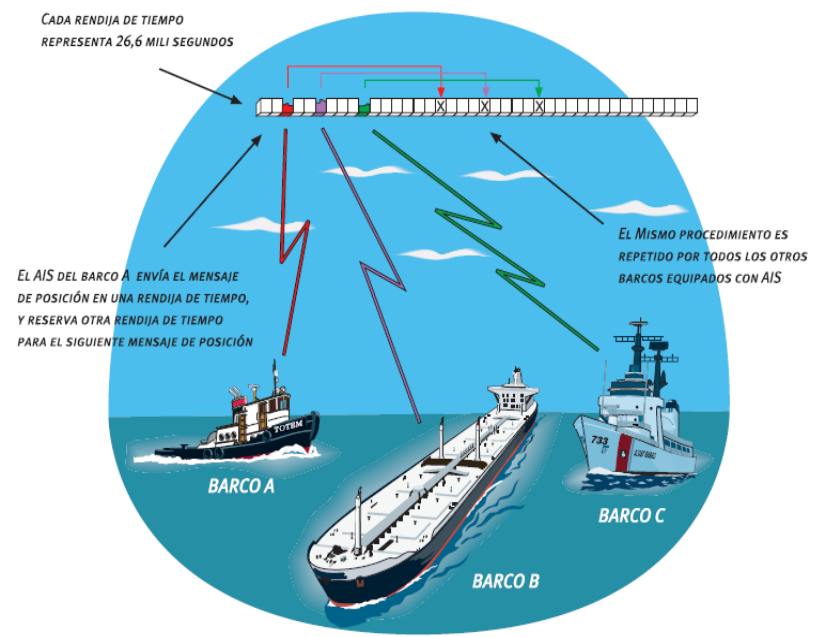

Figura 2. Esquema de compartición de tiempo SOTDMA [2]

Existen varias clases de dispositivos, cada uno diseñado para cumplir diferentes requisitos: Clase A, Clase B, Receptores, AtoN, SART y Estaciones Base.

a) Clase $A$

Transpondedores diseñados para distancias grandes en mar abierto, principalmente buques grandes de viajes internacionales o intercontinentales, la OMI ha determinado qué tipo de buques deben instalar estos dispositivos. Algunas de las características principales que ofrecen los equipos de clase A son:

- Potencia de transmisión: $12.5 \mathrm{~W}$ 
- Opciones de conectividad exhaustivas

- Pantalla para visualización de objetivos

- Opción de un pequeño teclado

- Amigable con el ambiente

- Tiempo de transmisión flexible dependiendo del viaje

- Transmisión mejorada para evitar la pérdida de datos a grandes distancias

\section{b) Clase $B$}

Transpondedores diseñados para embarcaciones menores como las de pesca, caracterizados por su tamaño y su baja potencia de transmisión.

Debido a esto tienen menor alcance sin embargo el tiempo de transmisión mejora notablemente al reducirse en comparación con los dispositivos de clase A. Algunas de las características de los equipos de clase B son:

- Potencia de transmisión: $2 \mathrm{~W}$

- Múltiples salidas para diferentes pantallas

- Puede poseer más de un transmisor, mismo que puede ser multiplexado para VHF DSC radio con un splitter.

\section{c) Receptores}

Diseñados para hacer monitoreos de tráfico sin ser detectados. Es capaz de leer señales AIS emitidas por cualquier clase de equipo que se encuentre dentro del rango de alcance; permite graficar estos objetivos en una pantalla, que puede ser incorporada o externa, sin enviar ningún tipo de información haciéndolo invisible ante los demás equipos. Posee múltiples salidas para adaptarlo a diferentes displays o sistemas. Posee puertos USB, NMEA 0183, NMEA 2000 o wifi.

d) AtoN

Diseñados específicamente para ayuda a la navegación, generalmente para aumentar el rango de alcance de las estaciones fijas de monitoreo o control. Estos equipos utilizan dos tipos de transmisión: RATDMA (Random Access Time Division Multiple Access) y FATDMA (Fixed Access Time Division Multiple Access). RATDMA es usado para expandir el alcance de estaciones fijas debido a su confiabilidad, esto es, como repetidores de las señales de las estaciones móviles. FATDMA es usado para los mensajes de ayuda a la navegación. Los principales contenidos del mensaje AIS son:

- Tipo de ayuda a la navegación

- Nombre de ayuda a la navegación

- Posición

- Indicador de exactitud de posición
- Indicador RAIM (Monitorización de la Integridad Autónoma del Receptor)

- Indicador fuera de posición

- Tipo de dispositivo de determinación de posición

- Dato horario

- Dimensión de la ayuda a la navegación y referencia para la posición

- 8 bits reservados para uso de la autoridad regional o local

- Señal virtual de ayuda a la navegación

e) SART

Diseñado para situaciones de emergencia, es pequeño, portátil y práctico. Permite localizar embarcaciones enviando la posición, identificación y velocidad. Una vez activados envían este mensaje de alerta de manera constante hasta que el equipo sea desactivado de manera manual o por falta de batería.

\section{f) Estaciones Base}

Diseñadas para proveer un enlace entre las estaciones móviles dentro de rango y un centro de comando nocostero. Ayudan a crear una red costera conectada con un único centro de control que no necesariamente estará ubicado en la costa, proveyendo de información de los AtoNs y de las estaciones móviles en los buques. Permite un control sobre el mapa de slots utilizando FATDMA para reservar slots para los AtoNs, también posee puertos de salida para conectar pantallas [3].

Para conexiones de dispositivos externos de salida o de entrada los dispositivos AIS cuentan con puertos USB, NMEA 0183, NMEA 2000 y wifi.

La versión de USB depende del equipo, así como el tipo de conector USB que utilice; los más actuales tienen conectores tipo B mini. Se puede utilizar el puerto USB para conectar dispositivos de salida como displays.

NMEA 0183 es una especificación que combina electricidad y datos, diseñada para aparatos electrónicos marinos [4]; permite conectar dispositivos externos de entrada o de salida.

NMEA 2000 es una norma de comunicación diseñada como mejora de la comunicación NMEA 0183.

El wifi es el protocolo de comunicación inalámbrica que permite conectar el equipo AIS con cualquier dispositivo que posea la tecnología wifi. Utilizado generalmente para tener la información de los equipos AIS en dispositivos móviles como los smartphones o tablets, además de permitir usar protocolo IP y conectar el equipo a internet mediante un router wifi. 


\section{Descripción del sistema propuesto}

Ante la evidente problemática de la delincuencia en altamar existente en Ecuador se propone un sistema de monitoreo de embarcaciones con tecnología AIS. Este sistema consta de 3 etapas: transmisor, repetidor, receptor; estas se muestran en la figura 3.

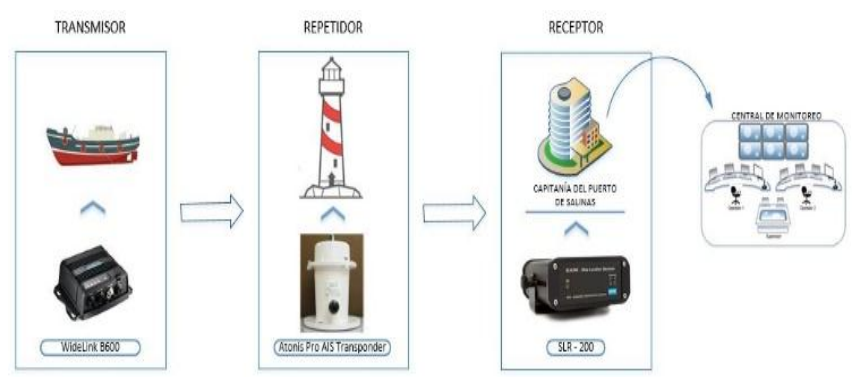

Figura 3: Estructura general de la red

Transmisores para enviar la ubicación exacta de las embarcaciones de pesca artesanal hacia la costa y repetidores ubicados estratégicamente para retransmitir la señal hacia el receptor colocado en la central de monitoreo para captar todas las señales AIS dentro del rango.

Se realizó el respectivo estudio para determinar la ubicación óptima para los repetidores y el receptor, tomando en consideración el alcance promedio de los dispositivos de la tecnología AIS y aprovechando los recursos existentes tales como infraestructura, energía eléctrica y equipos de cómputo.

\subsection{Etapa de Transmisión}

En esta etapa se obtiene la ubicación exacta de las embarcaciones por medio de la antena GPS que posee el equipo transceiver; con estas coordenadas se genera una trama que contiene, además de la ubicación, un ID propio del dispositivo (que puede ser programado internamente en el equipo) para identificar a cada embarcación, algunos datos de funcionamiento del dispositivo que se envían automáticamente para otras funcionalidades existentes y un bit de alerta que cambia de estado con un botón externo de pánico conectado a través de NMEA 0183.

Se utilizará el color gris para conectar dicho botón, configurando adecuadamente el dispositivo, cuando este se presione el bit de alerta cambiará a 1; el esquema de conexiones del puerto se muestra en la figura 4.

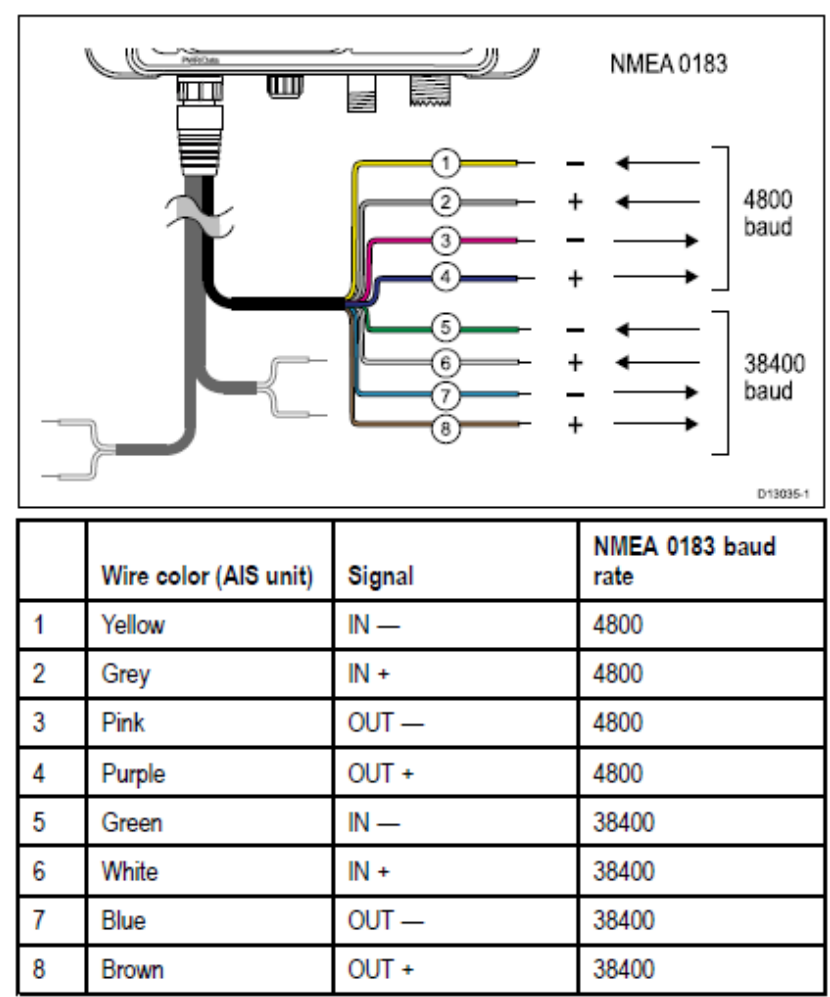

Figura 4. Diagrama de conexiones NMEA 0183

Esta trama es enviada por el canal AIS1 hacia la costa para ser captada por el repetidor.

En base a los requerimientos, en esta etapa del sistema se debe instalar un dispositivo clase $\mathrm{B}$ con las siguientes características:

- Bajo consumo de potencia

- Trabajar en el rango de frecuencias de ambos canales AIS1 y AIS2

- Portátil

- Tamaño pequeño

- Ancho de banda de $25 \mathrm{KHz}$

- $\quad$ Potencia de salida $\geq 5 \mathrm{~W}$

Se sugiere un equipo similar al WideLink B600 de AMEC. Cabe mencionar que las características de recepción del equipo a escoger son irrelevantes en esta etapa en particular.

Los transceiver serán instalados uno en cada embarcación registrada; debido al tamaño de las mismas es prioritario que el dispositivo seleccionado cumpla con el requerimiento de tamaño pequeño, ya que lo ideal es que sea instalado en una parte de la embarcación de pesca artesanal donde no sea visible para los delincuentes.

\subsection{Etapa de Repetición}

En esta etapa la señal enviada por las embarcaciones es recibida por el transpondedor colocado en la costa y reenviada por el canal AIS2 hacia el receptor. 
Como el repetidor estará ubicado en la costa los requerimientos para el dispositivo son diferentes que el de las embarcaciones, para esta etapa del sistema se debe instalar un dispositivo de clase A con las siguientes características:

- Trabajar en el rango de frecuencias de ambos canales AIS1 y AIS2

- Poseer al menos un receptor y un transmisor

- Ancho de banda de $25 \mathrm{KHz}$

- $\quad$ Potencia de salida $\geq 12.5 \mathrm{~W}$

- Nivel de sensibilidad $<-112 \mathrm{dBm}$

Se sugiere un equipo similar al Atonis Pro AIS Transponder de Automatic Power Inc.

Debido al área que se espera cubrir se debe instalar el transpondedor en una zona alta, en la parroquia de Anconcito una de las zonas más altas es el Faro. El Faro de Anconcito (Ubicación: Lat. $2^{\circ} 19^{\prime} 36^{\prime}$ S Long. $80^{\circ} 53$ '30" W) se encuentra a 51 metros sobre el nivel del mar (m.s.n.m.) y posee una altura 8 metros. La estructura existente no cuenta con instalaciones eléctricas, pero es factible el uso de un panel solar para la alimentación del dispositivo.

Para extender la cobertura y poder cubrir mejor el área crítica se escogió el Faro Santa Elena (Ubicación: Lat. $2^{\circ} 11$ ' 12.7'" S Long. $80^{\circ} 59^{\prime} 51.2^{\prime}$ W) que se encuentra a 95 m.s.n.m. y posee una altura de 12 metros. Estas instalaciones cuentan con una infraestructura civil en la parte inferior del faro y energía eléctrica.

En la figura 5 se muestran los círculos de la cobertura esperada con los dispositivos transpondedores en las ubicaciones mencionadas.

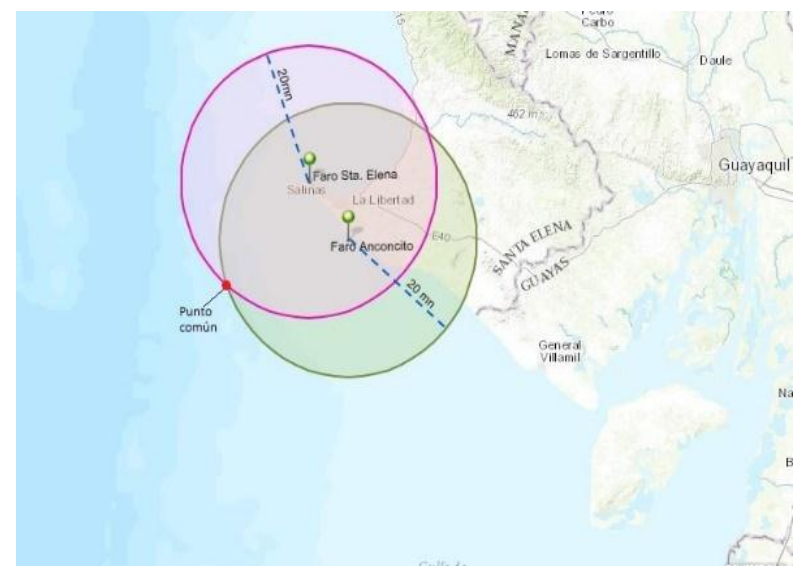

Figura 5. Ubicaciones de las estaciones repetidoras

La señal enviada por las embarcaciones será captada por la estación costera más cercana. En la figura 5 se observa que existe un traslape entre las coberturas de ambas estaciones; sin embargo los equipos seleccionados pueden ser configurados para que solo una de los dos repita el mensaje con una característica llamada Geographical Filtering. Esta es una particularidad que permite verificar la ubicación de la que proviene la trama y reenviarla o descartarla según los parámetros de configuración; para este sistema el transceiver del faro Santa Elena será configurado para descartar la trama si proviene de la zona de traslape mientras que el equipo de la estación costera del Faro Anconcito reenviará todas las tramas que reciba, de esta forma las zonas de cobertura resultantes sin traslape son mostradas en la figura 6 .

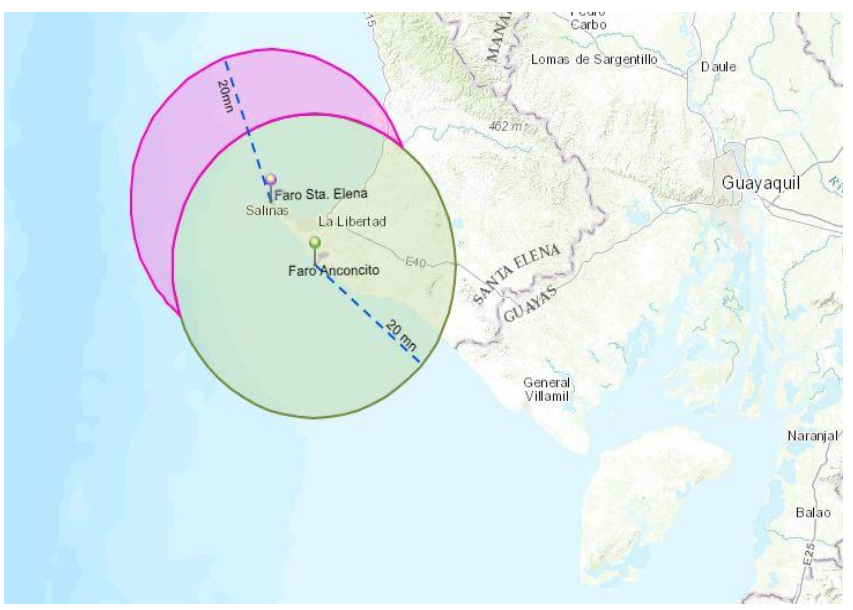

Figura 6. Cobertura de recepción de los dispositivos en el Faro Anconcito y el Faro Santa Elena sin traslape.

\subsection{Etapa de recepción}

En esta etapa se recibe, en la central de monitoreo, las señales retransmitidas por los repetidores. Para esto es necesario un dispositivo receptor y un equipo de cómputo con un software de monitoreo adecuado a las necesidades del sistema.

Dada la ubicación de este equipo los requerimientos son diferentes de las otras dos etapas, para esta etapa se recomienda usar un receiver con las siguientes características:

- Trabajar en el rango de frecuencias de ambos canales AIS1 y AIS2

- Nivel de sensibilidad $<-112 \mathrm{dBm}$

- Poseer al menos un receptor

Se sugiere un equipo similar al SLR-200 AIS Receiver de Automatic Power Inc.

La central de monitoreo será ubicada en la Capitanía del Puerto de Salinas que cuenta con infraestructura y equipos de cómputo adecuados, además de personal capacitado las 24 horas del día, los siete días de la semana. En la figura 7 se observa la ubicación de la central de monitoreo. 


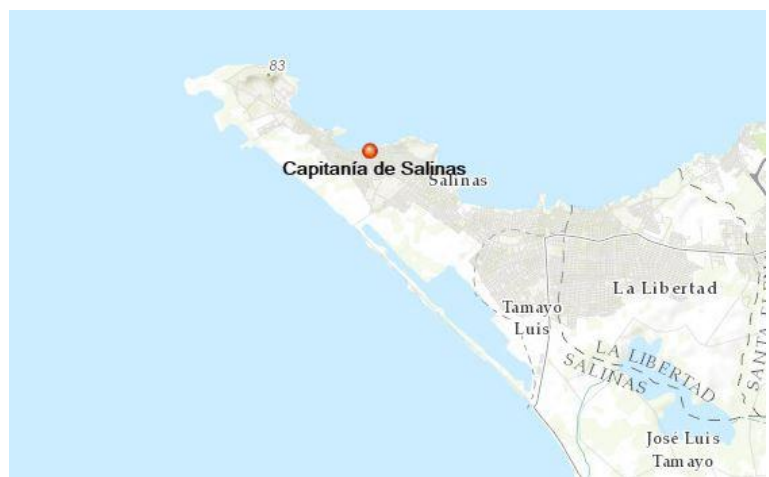

Figura 7. Ubicación de la central de monitoreo

El software de monitoreo debe contar con las siguientes características:

- Decodificar todos los mensajes AIS

- Decodificar cualquier Mensaje de Aplicación Específica

- Calcular la estadística de disponibilidad

- Permitir personalizar la apariencia para el mensaje 6 y 8 de cada estación

La correcta instalación del software adecuado permitirá observar en el monitor la ubicación de cada embarcación artesanal con su respectivo ID y una notificación de alerta en caso de presionarse algún botón de pánico.

Se sugiere un software similar a iNav AtoN Monitoring Software de Automatic Power Inc. que cumple con los requerimientos del sistema.

\subsection{Diseño del sistema}

Se realizó el análisis de propagación utilizando el software Radio Mobile para verificar que es posible realizar el enlace en el peor de los casos (a $20 \mathrm{mn}$ de las estaciones). Para facilidad de simulación el transmisor se colocó en el punto común entre los círculos de cobertura mostrados en la figura 5 (ubicación: $2^{\circ} 26^{\prime} 19^{\prime \prime} \mathrm{S} 81^{\circ} 12^{\prime} 45^{\prime}$ 'W) con la altura de la antena de 1 metro y potencia de $5 \mathrm{~W}$.

El análisis realizado entre el transmisor y la estación costera del Faro Anconcito mostró que el enlace es posible; sin embargo, el nivel de potencia de recepción es - 100 dBm y dado que la sensibilidad del equipo es $-112 \mathrm{dBm}$ el margen de desvanecimiento del enlace es $12 \mathrm{~dB}$, lo cual asegura un enlace viable pero crítico ya que lo ideal sería tener 15-20 dB de acuerdo a la Recomendación UIT-R P.530-13. Al tener un nivel crítico de recepción el enlace se encuentra expuesto a ser fácilmente afectado por condiciones climáticas poco favorables, sin embargo esta situación se puede mejorar simplemente colocando la antena en una torre de 20 metros junto al faro, con lo cual la potencia de recepción es de -96.6 $\mathrm{dBm}$ y el margen de enlace sería de $15.4 \mathrm{~dB}$. La frecuencia utilizada para este enlace fue el canal AIS1 - 161.975 MHz.
Para el enlace entre el trasmisor y la estación costera del Faro Santa Elena se utilizó la frecuencia AIS1 - 161.9751 $\mathrm{MHz}$. Se observó que el enlace si es posible; la potencia de recepción es $-94.4 \mathrm{dBm}$ y la sensibilidad del equipo es de $-112 \mathrm{dBm}$ lo que da un margen de enlace de $17.6 \mathrm{~dB}$, esto asegura no solo un enlace viable sino también robusto ante interferencias y condiciones climáticas adversas. Se puede optimizar el margen de enlace instalando el equipo transceiver en la torre de 30 metros que se encuentra junto al faro. En la figura 8 se muestran las coberturas de recepción de la central de monitoreo y las estaciones costeras.

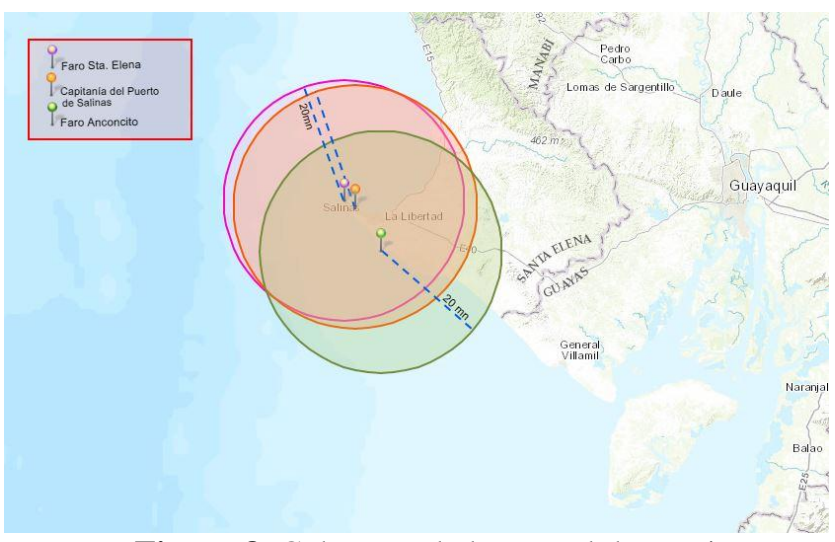

Figura 8. Cobertura de la central de monitoreo

Se realizó también el análisis del enlace entre la estación costera del Faro Anconcito y la estación de monitoreo en la Capitanía del Puerto de Salinas, en este se observó que el nivel de recepción obtenido es de $-66.68 \mathrm{dBm}$ y dado que la sensibilidad del equipo es de $-112 \mathrm{dBm}$ se obtiene un margen de enlace de $45.2 \mathrm{~dB}$, por lo que se puede asegurar un enlace completamente estable y resistente ante adversidades. Para este enlace se utilizó el canal AIS2 - $162.025 \mathrm{MHz}$ y así evitar interferencias perjudiciales con las trasmisiones de los barcos.

En el enlace entre la estación costera del Faro Santa Elena y la central de monitoreo se obtuvo un nivel de potencia de recepción de $-42.5 \mathrm{dBm}$, con una sensibilidad del equipo de $-112 \mathrm{dBm}$ se tiene un margen de enlace de $69.5 \mathrm{~dB}$, que es un nivel óptimo y asegura un enlace robusto ante interferencias. En este enlace también se utilizó el canal AIS2 - 162.0251 MHz ya que, como se observa en la figura 5, la cobertura del receptor de la central de monitoreo se traslapa con las coberturas de las estaciones costeras y de usarse el canal AIS1 esto puede generar interferencias perjudiciales.

\section{Resultados y Discusión}

El principal resultado esperado luego de la implementación del sistema propuesto es mejorar la seguridad de los pescadores y de sus herramientas de trabajo, tanto los motores como los implementos de pesca; con esto se 
beneficia la comunidad pesquera y toda la población de Anconcito.

El sistema de monitoreo presentado en este estudio tiene como propósito evitar los asaltos a los pescadores, pero principalmente evitar las pérdidas humanas. Este sistema permite llevar un control permanente y constante de las embarcaciones que se encuentran en altamar y en caso de ser asaltados pueden ser fácilmente asistidos por la Comandancia de Guardacostas, bien sea para poder evitar el asalto y capturar a los hampones o para el procedimiento de Search And Rescue.

Si el sistema es integrado a la base de datos del SIGMAP (Sistema de Gestión Marítimo y Portuario) se puede llevar un registro de los asaltos ocurridos; con estos datos es posible generar un patrón y proponer rutas de patrullaje para las unidades de guardacostas; esto permitirá que las autoridades puedan acudir inmediatamente al lugar de los hechos y tener más probabilidades de atrapar a los asaltantes.

Previamente se han instalado otros sistemas de control que han resultado ineficaces y esto podría resultar en un rechazo por parte de los pescadores hacia nuestro sistema de monitoreo; para evitar esto se hará una campaña enfocada principalmente en la tecnología AIS y sus ventajas.

Una de las principales ventajas de AIS por sobre la tecnología implementada anteriormente (sistema de posicionamiento satelital) es el tamaño de los dispositivos. AIS ofrece equipos pequeños y prácticos, fáciles de instalar en partes de la embarcación donde no sea visible, haciendo más difícil para los asaltantes arrancar los equipos y destruirlos.

Al implementar este sistema se espera que el índice de atracos disminuya y con ello mejore la economía de la población. En un muestreo realizado para el análisis de la situación en Anconcito se observó que los atracos representan grandes pérdidas económicas para los pescadores. Al mejorar la economía de los pescadores es posible que estos inviertan más dinero en sus implementos de trabajo o en el mantenimiento de sus motores y embarcaciones de pesca artesanal. Todo esto conllevaría a la activación de la economía de Anconcito.

El sistema propuesto está considerado para tener un alcance máximo de $20 \mathrm{mn}$; sin embargo es posible mejorar esta cobertura; a continuación se presentan algunas propuestas:

\section{a) Repetidores en las unidades guardacostas}

Se pueden instalar dispositivos repetidores en las lanchas de guardacostas y gracias a las rutas extendidas de patrullaje estas servirían para extender el área de cobertura. En la figura 9 se muestra cómo podría aumentar el alcance del sistema.

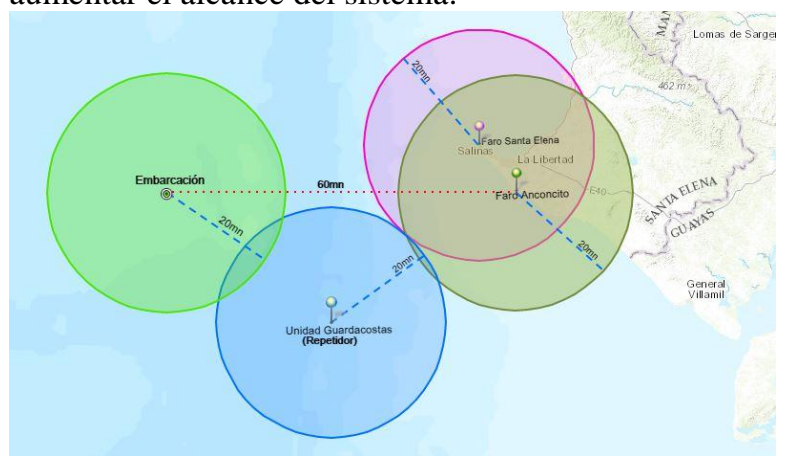

Figura 9. Extensión de cobertura con un repetidor en la unidad guardacostas

El círculo azul de la figura 9 representa el área que se extiende la cobertura del sistema si una lancha guardacostas se encuentra en esa ubicación.

\section{b) Buques con dispositivos AIS como repetidores}

Para los buques intercontinentales y los buques con arqueo bruto superior a 500TRB el estándar AIS es obligatorio; por lo tanto es posible usar los dispositivos de esos buques como repetidores para extender la cobertura del sistema. Para esto es necesario un estudio de las rutas fijas de estos buques y así establecer las posibles coberturas y los horarios. De llegar a realizarse, esto podría ayudar a variar las rutas guardacostas también propuestas y ampliar el área de cobertura aún más.

\section{c) Repetidores en boyas}

En un estudio posterior es posible hacer un análisis de las ubicaciones de boyas oceánicas para mejorar el alcance. Actualmente existen dos boyas oceánicas: en la provincia de Manta (Ubicación: Lat. $1^{\circ} 12$ ' 36" S Long. $81^{\circ} 48^{\prime} 00^{\prime}$ 'W) y en la provincia de Esmeraldas (Ubicación: Lat. 0³4'12" N Long. 81 16’12"). Para el sistema planteado en el presente proyecto estas boyas se encuentran muy lejos, sería necesario colocar otra boya con un repetidor a $37 \mathrm{mn}$ del faro Santa Elena; esto aumentaría el alcance a $100 \mathrm{mn}$ aproximadamente como se observa en la figura 10. 


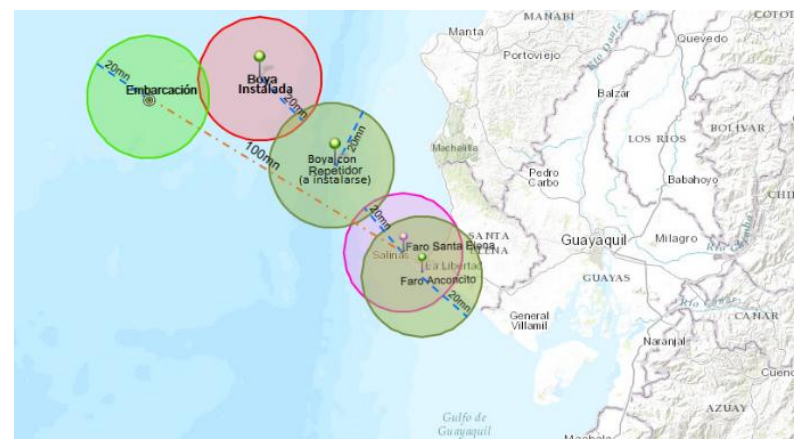

Figura 10. Extensión de cobertura con dos boyas repetidoras

La empresa Automatic Power Inc. ofrece boyas con repetidores Atonis integrados como se muestra en la figura 11.

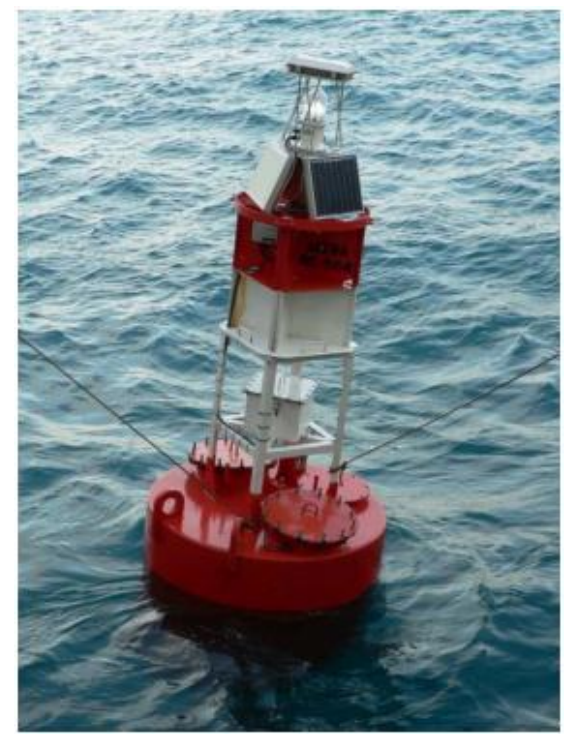

Figura 11. Boya con repetidor Atonis integrado [5]

\section{d) AIS Satelital}

La tecnología AIS permite configurar el modo de transmisión satelital colocando la antena adecuada, como la Inmarsat D+. Con un sistema de comunicación satelital se puede extender la cobertura del sistema de monitoreo a todo el mar territorial ecuatoriano; cabe mencionar que en ese caso no serían necesarios los repetidores en los faros ya que se puede recibir directamente la información en la central de monitoreo de la Capitanía de Salinas.

\section{Conclusiones}

- De implementarse el sistema de monitoreo propuesto en este proyecto la economía del pueblo de Anconcito mejoraría notablemente ya que disminuiría la delincuencia en altamar, con esto se reducen las pérdidas económicas producto de los asaltos, además del tiempo sin actividad cuando las embarcaciones de pesca artesanal se quedan sin motor.

- $\mathrm{Si}$ se adiciona el sistema de monitoreo con tecnología AIS para lanchas de pesca artesanal se asegura la vida de los pescadores, ya que se podría evitar que los incidentes pasen a mayores y se pierdan vidas humanas como consecuencia de quedar a la deriva sin motor y sin comunicación.

- La tecnología AIS ofrece información completa y detallada sobre cada una de las embarcaciones para poder tener así un mayor control sobre estas. AIS además permite incorporar en la trama que se emite diferentes elementos con fines específicos como códigos o señales que ayuden en el proceso de búsqueda y rescate.

- AIS ofrece equipos con características particulares y adecuadas para cada tipo de sistema, permitiendo diseñar la red acorde a las circunstancias y necesidades de la misma.

\section{Referencias}

Estándares:

[1] Technical characteristics for an automatic identification system using time-division multiple access in the VHF maritime mobile band, Recommendation ITU-R M.1371-4, Abr. 2010.

[2] "Información AIS", en Guía de las Ayudas a la Navegación Marítima, 4ta ed., Ed. Ente Público Puertos del Estado, España, 2004, pp. 115, 118-119

[3] "All About AIS" [online], 2012 Disponible en:http://www.allaboutais.com/index.php/en/technical -info/transmission-types

[4] AIS350/650 Installation Instructions, Raymarine UK Ltd., 2014.

[5] "Pharos Marine Automatic Power" [online], 2014 Disponible en: http://www.automaticpower.com/products/BL-620BUOY-013114B.pdf 